program orders. Unless, therefore, there are some orders in the store at the beginning of the computation, nothing can be taken in through the input, and the machine cannot start. For this reason, there is a sequence of orders, known as initial orders, permanently wired onto a set of uniselectors (rotary telephone switches). These orders can be transferred to the store by pressing a button.

There is considerable latitude in the choice of the initial orders, although once they have been wired onto the uniselectors, it is not easy to change them. The initial orders used in the EDSAC at present enable orders punched in the following form to be taken in from the tape. First a letter indicating the function is punched, then the numerical part of the order in decimal form, and finally the letter $\mathrm{F}$ or $\mathrm{D}$ indicating, respectively, that the order refers to a long or a short number. If the order has no numerical part, it is punched simply as a letter followed by F. Under the control of the initial orders the machine converts the numerical part of the order to binary form and assembles the order with the function digits and the numerical digits in their correct relative positions.

The University Mathematical Laboratory

M. V. WILKES

W. RENWICK

Cambridge, England

${ }^{1} \mathrm{M}$. V. WiLkes, "The design of a practical high-speed computing machine. The EDSAC," R. Soc., London, Proc., v. 195, 1948, p. 274-279.

M. V. WILKES, "Programme design for a high-speed automatic calculating machine," $J n$. of Sci. Inst. and Phys. in Industry, v. 26, 1949, p. 217-220.

M. V. WILKES \& W. RENWICK, "An ultrasonic memory unit for the EDSAC," Electronic Engineering, v. 20, 1948, p. 208-213.

\title{
Convergence Rates of Iterative Treatments of Partial Differential Equations
}

1. Introduction. The development of high-speed digital computers ${ }^{1}$ has made feasible the numerical solution by iterative methods of some partial differential equations. The convergence rates of several such iterative methods are estimated here. It is found that with the familiar elementary iterative methods some quite simple problems require prohibitive computational labor.

The iterative methods here considered are related to the various forms of the SOUTHWELL "relaxation method"2,4 in that they involve successively applied local corrections to improve an approximate solution. However, these iterative methods are routinized in conformity with the requirements of automatic computers while the relaxation method is flexible and depends in an essential way on the skill of its practitioners.

2. Reduction to Finite Difference Form. The iterative methods of successive approximation considered here are, like the relaxation method, not directly applicable to partial differential equations (and associated boundary conditions) but only to the finite difference approximations to them derived in the customary way. ${ }^{3}$ For example, the LAPLACE equations, $\Delta \phi=0$, applicable within a region $R$ in the $x, y$ plane may be approximated by the 
difference equation:

(1) $L \phi(x, y) \equiv \phi(x+h, y)+\phi(x-h, y)+\phi(x, y+h)$

$$
+\phi(x, y-h)-4 \phi(x, y)=0
$$

applied at those points $(x=j h, y=k h)$, of a rectangular lattice which lie within the region $R$. We are here concerned only with the rate of convergence to the solution of this set of algebraic equations and not with closeness of that approximation to the solution of the differential equation. In the following we will use this difference equation, applied within a rectangular region, as an illustrative example for each of the iteration methods considered. We denote the value assigned to $\phi(j h, k h)$ in the $n$th stage of iteration by $\phi_{j, k}^{n}$. The limit approached with increasing $n$ we denote by $\phi_{j, k}$. For definiteness we take $R$ to be the rectangular region

$$
R=\{j=0,1, \cdots, p, \quad k=0,1, \cdots, q\} ;
$$

on the boundary of this region we assign fixed values of $\phi$. Thus

$$
\begin{aligned}
& \phi_{j, k}^{n}=\phi_{j, k}=b_{j, k} \quad \text { for } \quad j=0 \text { or } p, \quad k=0 \text { or } q . \\
& L \phi_{j, k} \equiv \phi_{j-1, k}+\phi_{j+1, k}+\phi_{j, k-1}+\phi_{j, k+1}-4 \phi_{j, k}=0 \\
& \text { for } \quad \begin{aligned}
j & =1,2, \cdots, p-1 \\
k & =1,2, \cdots, q-1
\end{aligned}
\end{aligned}
$$

In all of the methods here considered $\phi_{j, k}^{0}$ is a first (guessed) approximation to $\phi_{j, k}$. Each of the succeeding approximations, $\phi^{1}, \phi^{2}, \cdots$, is calculated on the basis of its predecessors (or immediate predecessor) by some process which guarantees the convergence of $\phi^{n}$ to $\phi$.

3. The Richardson Method. Following Shortley, Weller and Fried ${ }^{4}$ we term the most elementary of the familiar iterative procedures the RICHARDSON method. ${ }^{5}$ In this method the correction process applied to each $\phi^{n}$ consists of the addition of a positive multiple of $L \phi^{n}$ (for each interior point). Thus

$$
\begin{aligned}
\phi_{j, k}^{n+1} & =\phi_{j, k}^{n}+\alpha L \phi_{j, k}^{n} \equiv \phi_{j, k}^{n} \\
& +\alpha\left[\phi_{j-1, k}^{n}+\phi_{j+1, k}^{n}+\phi_{j, k-1}^{n}+\phi_{j, k+1}^{n}-4 \phi_{j, k}^{n}\right] \text { (interior points) } \\
\phi_{j, k}^{n+1} & =\phi_{j, k}^{n}=b_{j, k} \\
& \text { (boundary points). }
\end{aligned}
$$

The error at each stage we denote by $\epsilon_{j, k}^{n}$,

$$
\epsilon_{j, k}^{n} \equiv \phi_{j, k}^{n}-\phi_{j, k} .
$$

By substitution in (3) we obtain the error recurrence relation,

$$
\epsilon_{j, z}^{n+1}=\epsilon_{j, k}^{n}+\alpha L \epsilon_{j, k}^{n} \quad \text { (interior points), }
$$

since $L \phi_{j, k}=0$; or more briefly

$$
\begin{aligned}
\epsilon^{n+1} & =(1+\alpha L) \epsilon^{n} & & \text { (interior points) } \\
& =0 & & \text { (boundary points). }
\end{aligned}
$$

The most familiar form of the Richardson method is that obtained by setting $\alpha=\frac{1}{4}$. Then equation (3) reduces to

$$
\begin{array}{rlr}
\phi_{j, k}^{n+1} & =\frac{1}{4}\left[\phi_{j-1, k}^{n}+\phi_{j+1, k}^{n}+\phi_{j, k-1}^{n}+\phi_{j, k+1}^{n}\right] \quad \text { (interior points) } \\
& =b_{j, k} \quad \text { (boundary points). }
\end{array}
$$


As shown below, this form is not only numerically more convenient, by reason of the disappearance of $\phi_{j, k}^{n}$ from the right member, but is also in one sense the most efficient form.

To determine the convergence rate of this process we expand $\epsilon^{0}$ in the eigenfunctions of the operator $L$ subject to the boundary condition of (4). These are evidently, ${ }^{6}$

$$
\epsilon_{j, i}^{(r, s)}=\sin (\pi r j / p) \sin (\pi s k / q), \quad \begin{aligned}
& r=1,2, \cdots, p-1 \\
& s=1,2, \cdots, q-1 .
\end{aligned}
$$

The corresponding eigenvalue of $L$ we denote by $L_{(r, s)}$

$$
\begin{aligned}
L \epsilon_{j, k}^{(r, s)} & =\epsilon_{j-1, k}^{(r, s)}+\epsilon_{j+1, k}^{(r, s)}+\epsilon_{j, k-1}^{(r, s)}+\epsilon_{j, k+1}^{(r, s)}-4 \epsilon_{j, k}^{(r, s)} \\
& =[\sin (\pi r(j-1) / p)+\sin (\pi r(j+1) / p)-2 \sin (\pi r j / p)] \sin (\pi s k / q) \\
& +[\sin (\pi s(k-1) / q)+\sin (\pi s(k+1) / q)-2 \sin (\pi s k / q)] \sin (\pi r j / p) \\
& =[2 \cos (\pi r / p)-2] \sin (\pi r j / p) \sin (\pi s k / q) \\
& +[2 \cos (\pi s / q)-2] \sin (\pi r j / p) \sin (\pi s k / q) \\
& =[2 \cos (\pi r / p)+2 \cos (\pi s / q)-4] \epsilon_{j, k}^{(r, s)}=L_{(r, s) \epsilon_{j, k}^{(r, s)},}^{(\pi)}
\end{aligned}
$$

hence

$$
L_{(r, s)}=-4\left[\sin ^{2}(\pi r / 2 p)+\sin ^{2}(\pi s / 2 q)\right] .
$$

All of these eigenvalues are negative. The smallest and largest in magnitude belong to $r=s=1$ and to $r=p-1, s=q-1$, respectively. We denote these by $L_{0}$ and $L_{m}$.

$$
\begin{aligned}
L_{0}=-4\left[\sin ^{2}(\pi / 2 p)\right. & \left.+\sin ^{2}(\pi / 2 q)\right] \cong-\pi^{2}\left(p^{-2}+q^{-2}\right) \\
L_{m}=-4\left[\sin ^{2}(\pi(p-1) / 2 p)\right. & \\
& \left.\quad+\sin ^{2}(\pi(q-1) / 2 q)\right] \cong-8+\pi^{2}\left(p^{-2}+q^{-2}\right) .
\end{aligned}
$$

The eigenfunctions of $L$ are also eigenfunctions of the iteration operation, $K \equiv(1+\alpha L)$, corresponding to the eigenvalues

$$
K_{(r, s)}=1+\alpha L_{(r, s)} \text {. }
$$

Each error-eigenfunction component is multiplied by its $K_{(r, s)}$ at each iteration. Thus if

$$
\epsilon_{j, k}^{0}=\sum_{r, s} a_{r, 8} \epsilon_{j, k}^{(r, s)}
$$

then

$$
\epsilon_{j, k}^{n}=\sum_{r, s} a_{r, s} \epsilon_{j, k}^{(r, s)} K_{(r, s)}^{n}
$$

With increasing $n$ those components, $\epsilon^{(r, s)}$, for which

$$
\left|K_{(r, s)}\right|<1
$$

diminish in importance, the more rapidly the smaller is $\left|K_{(r, s)}\right|$. This iteration method is convergent (in general) only if (11) is satisfied for all $r, s$ : The ultimate convergence rate is determined by the maximum magnitude of $K$,

$$
K^{*} \equiv \max _{(\tau, s)}\left|K_{(r, s)}\right| .
$$

Since by $(9) K_{(r, s)}$ lies in the range

$$
K_{m} \equiv 1+\alpha L_{m} \leqslant K_{(r, s)} \leqslant 1+\alpha L_{0} \equiv K_{0}
$$


the equality signs holding for $(r, s)=(1,1)$ and $(p-1, q-1), K$ is determined only by these extremes,

$$
K^{*}=\max \left\{\left|K_{m}\right|, \quad\left|K_{0}\right|\right\} .
$$

As $\alpha$ increases from zero, $K_{0}$ drops slowly from unity, $K_{m}$ drops rapidly from unity. Thus $K^{*}=K_{0}>0$ so long as $K_{0} \geqslant-K_{m}$. For greater values of $\alpha$, $K^{*}=-K_{m}$, hence $K^{*}$ then rises with increasing $\alpha$. The smallest $K^{*}$ (hence the most rapid convergence) occurs where

hence for

$$
K_{0}=1+\alpha L_{0}=-K_{m}=-\left(1+\alpha L_{m}\right),
$$

$$
\alpha=-2 /\left(L_{0}+L_{m}\right)=\frac{1}{4} .
$$

For this optimum $\alpha$-value the error eigenfunctions of longest and shortest "wavelength," $\epsilon^{(1,1)}$ and $\epsilon^{(p-1, q-1)}$, decay at the same rate

$$
\begin{aligned}
& K^{*}=1-\sin ^{2}(\pi / 2 p)-\sin ^{2}(\pi / 2 q) \\
& \quad=\frac{1}{2}[\cos (\pi / p)+\cos (\pi / q)] \cong 1-\pi^{2}\left(p^{-2}+q^{-2}\right) / 4
\end{aligned}
$$

(the short wavelength error alternating in sign) while other errors decay more rapidly.

The numerical processes carried out in this iterative approach to the solution of a Laplace equation may also be regarded as the successive steps in the solution of the heat flow equation,

$$
\begin{aligned}
\frac{\partial \phi}{\partial t} & =\Delta \phi & & \text { (interior) } \\
\phi & =b & & \text { (boundary). }
\end{aligned}
$$

The parameter, $\alpha$, then plays the rôle of

$$
\frac{\Delta t}{(\Delta x)^{2}}=\frac{\Delta t}{(\Delta y)^{2}}=\frac{\Delta t}{h^{2}}
$$

This method of solution of the heat flow equation is unstable ${ }^{7}$ for $\alpha$ appreciably greater than $\frac{1}{4}$, i.e., for a time interval appreciably greater than $\frac{1}{4}(\Delta x)^{2} \equiv \frac{1}{4}(\Delta y)^{2}$. For smaller $\alpha$-values the solution approaches asymptotically a stationary form, hence one satisfying the Laplace equation.

The optimum property of $\alpha=\frac{1}{4}$ in the Richardson treatment of the Laplace equation is not peculiar to the boundary conditions here considered. If $(L+4)$ has an eigenfunction, $\epsilon_{j k}^{\prime}$, belonging to the eigenvalue $\left(L^{\prime}+4\right)$,

$$
\left(L^{\prime}+4\right) \epsilon_{j k}^{\prime}=\epsilon_{j-1, k}^{\prime}+\epsilon_{j+1, k}^{\prime}+\epsilon_{j, k-1}^{\prime}+\epsilon_{j, k+1}^{\prime},
$$

then $\epsilon^{\prime \prime} \equiv(-1)^{i+k} \epsilon^{\prime}$, if it is consistent with the boundary conditions, is an eigenfunction belonging to the eigenvalue $\left(L^{\prime \prime}+4\right)=-\left(L^{\prime}+4\right)$,

$$
\begin{gathered}
\left(L^{\prime \prime}+4\right) \epsilon_{j, k}^{\prime \prime}=-\left(L^{\prime}+4\right) \epsilon_{j, k}^{\prime \prime}=\epsilon_{j-1, k}^{\prime \prime}+\epsilon_{j+1, k}^{\prime \prime}+\epsilon_{j, k-1}^{\prime \prime}+\epsilon_{j, k+1}^{\prime \prime} \\
L^{\prime \prime}=8-L^{\prime} .
\end{gathered}
$$

If the boundary conditions permit this reversal, $\epsilon^{\prime} \rightarrow \epsilon^{\prime \prime}$, then for each eigenvalue, $L_{i}$, there also occurs the eigenvalue $-8-L_{i}$. Then $L_{0}+L_{m}=-8$ leading to optimum convergence for $\alpha=\frac{1}{4}$.

For other linear equations,

$$
L \phi=0 \text {, }
$$


where $L$ is the linear difference operator corresponding to the linear differential operator under consideration, the Richardson method,

$$
\phi^{n+1}=\phi^{n}+\alpha L \phi^{n},
$$

may be made convergent provided all of the eigenvalues of $L$ are of the same sign (and not zero).

The optimum convergence rate is given by

$$
\begin{aligned}
\alpha & =-2 /\left(L_{0}+L_{m}\right) \\
K^{*} & =1-\alpha L_{0}=1-2 \eta,
\end{aligned}
$$

where $\eta$ denotes the ratio,

$$
\eta=L_{0} /\left(L_{0}+L_{m}\right) .
$$

Here again the Richardson method is formally equivalent to the solution of a partial differential equation in one more variable,

$$
\frac{\partial \phi}{\partial t}=L \phi,
$$

the solution being carried to a sufficiently great $t$ to make the rate of change with $t$ negligible, hence also

$$
L \phi \cong 0 \text {. }
$$

4. The Liebmann Method. In the LiebmanN method ${ }^{8}$ a correction process like that of the Richardson method is applied to each of the lattice points in succession in a regular pattern. The $\phi$-value so corrected is used in all subsequent operations in that iteration step. It may thus be termed a "continuous substitution method." In its simplest form the lattice is scanned in the same direction along successive rows. Thus, as applied to the Laplace equation and boundary conditions described above, the Liebmann iteration process may be written,

$$
\begin{array}{rlrl}
\phi_{j, k}^{n+1} & =\phi_{j, k}^{n}+\alpha\left[\phi_{j-1, k}^{n+1}+\phi_{j+1, k}^{n}+\phi_{j, k-1}^{n+1}+\phi_{j, k+1}^{n}-4 \phi_{j, k}^{n}\right] \\
& =b_{j, k} & & \text { (interior points) } \\
& \text { (boundary points). }
\end{array}
$$

If $\alpha$ is again given the value $\frac{1}{4}$ this expression becomes

$$
\phi_{j, k}^{n+1}=\frac{1}{4}\left[\phi_{j-1, k}^{n+1}+\phi_{j+1, k}^{n}+\phi_{j, k-1}^{n+1}+\phi_{j, k+1}^{n}\right],
$$

thus $L \phi_{j, k}$ is brought to zero (momentarily) at each of the lattice points in succession. In this form the Liebmann procedure may be regarded as a very mechanical application of the relaxation method.

The iteration equation for the error

$$
\epsilon_{j, k}^{n} \equiv \phi_{j, k}^{n}-\phi_{j, k}
$$

is obtained by substitution in (19),

$$
\begin{array}{rlr}
\epsilon_{j, k}^{n+1} & =\epsilon_{j, k}^{n}+\alpha\left[\epsilon_{j-1, k}^{n+1}+\epsilon_{j+1, k}^{n}+\epsilon_{j, k-1}^{n+1}+\epsilon_{j, k+1}^{n}-4 \epsilon_{j, k}^{n}\right] \\
& =0 & \text { (interior points) } \\
& \text { (boundary points). }
\end{array}
$$


This error interation process can be written briefly as

$$
\epsilon^{n+1}=K(\alpha) \epsilon^{n},
$$

where $K(\alpha)$ is a linear operator depending on the parameter $\alpha$, but now not simply related to the Laplace (difference) operator, $L$. We again examine the spectrum of eigenvalues of $K(\alpha)$ and regard the greatest magnitude of these eigenvalues as a measure of convergence rate. If $K$ is an eigenvalue of (22) then its eigenfunction $\epsilon_{j, k}$ (we suppress indexing of the eigenfunctions and eigenvalues) must satisfy the following equation, obtained by substituting (22) in (21)

$$
\begin{aligned}
K \epsilon_{j, k} & =\epsilon_{j, k}+\alpha\left[K \epsilon_{j-1, k}+\epsilon_{j+1, k}+K \epsilon_{j, k-1}+\epsilon_{j, k+1}-4 \epsilon_{j, k}\right], \\
\epsilon_{0, k} & =\epsilon_{p, k}=\epsilon_{j, 0}=\epsilon_{j, q}=0 .
\end{aligned}
$$

We seek solutions of the form

$$
\epsilon_{j, k}=A^{i} \sin (\pi r j / p) \cdot B^{k} \sin (\pi s k / q) \quad \begin{aligned}
& r=1,2, \cdots, p-1 \\
& s=1,2, \cdots, q-1 .
\end{aligned}
$$

Substituting in (23) gives,

$$
\begin{aligned}
& (K-1+4 \alpha) A^{i} B^{k} \sin (\pi r j / p) \sin (\pi s k / q) \\
& =B^{k} \sin (\pi s k / q)\left[K A^{j-1} \sin (\pi r(j-1) / p)\right. \\
& \left.+A^{j+1} \sin (\pi r(j+1) / p)\right]+\alpha A^{j} \sin (\pi r j / p) \\
& \quad \times\left[K B^{k-1} \sin (\pi s(k-1) / q)+B^{k+1} \sin (\pi s(k+1) / q)\right] .
\end{aligned}
$$

To prevent the appearance of terms in $\cos (\pi r j / p)$ and $\cos (\pi s k / q)$ in the right member of (25) we must require

$$
A^{2}=B^{2}=K \text {. }
$$

Since multiplication of $A$ (or $B$ ) by -1 is equivalent to the replacement of $r$ by $p-r$ (or $s$ by $q-s$ ) we may, without loss of generality, take $A=B$. Equation (25) then becomes

or

$$
\left(A^{2}-1+4 \alpha\right)=2 \alpha A[\cos (\pi r / p)+\cos (\pi s / q)]
$$

$$
A^{2}-2 \alpha t A+(4 \alpha-1)=0 ; t \equiv[\cos (\pi r / p)+\cos (\pi s / q)] .
$$

The appearance of two values (in general) of $A$ for each $(r, s)$ would seem to give more than the $(p-1)(q-1)$ possible linearly independent error eigenfunctions; however, the replacement of $(r, s)$ by $(p-r, q-s)$ merely changes the sign of $t$, hence multiplies the two $A$-roots by -1 . Thus (24), $(26)$, and (27) define just the $(p-1)(q-1)$ linearly independent eigenfunctions required to form a complete set.

If we again take $\alpha=\frac{1}{4}$ the roots of (27) are

$$
A=0, \frac{t}{2} \text {. }
$$

The value $A=0$ corresponds to the complete removal of an isolated error occurring at $j=1$ or $k=1$. The other root gives

$$
K=A^{2}=\frac{1}{4} t^{2}=\frac{1}{4}[\cos (\pi r / p)+\cos (\pi s / q)]^{2} .
$$


The greatest eigenvalue occurs both for $(r=s=1)$ and for $(r=p-1$, $s=q-1)$, and is,

$$
K^{*}=\frac{1}{4}[\cos (\pi / p)+\cos (\pi / q)]^{2} \cong\left[1-\pi^{2}\left(p^{-2}+q^{-2}\right) / 4\right]^{2} .
$$

Thus in each iteration cycle the most resistant errors are reduced as much as in two cycles in the Richardson procedure. (This doubling of efficiency is qualitatively plausible since the numbers from which each $\phi_{j, k}^{n+1}$ is compounded are in the mean half as "old" in the Liebmann as in the Richardson procedure.)

5. The Extrapolated Liebmann Method. Since the eigenvalues, $K$, are all positive it seems plausible that increasing $\alpha$ should improve the efficiency of this process. The procedure so obtained we may term the "extrapolated Liebmann method." Examination of equation (26) shows that an improvement in convergence rate can be so obtained. For $\alpha>\frac{1}{4}$ the constant term is positive. We may therefore distinguish two ranges of $\alpha$, hence of the middle term. Where

$$
\alpha^{2} t^{2} \leqslant 4 \alpha-1 ; t^{2} \leqslant(4 \alpha-1) / \alpha^{2}
$$

the two roots of (26) are complex conjugates having the magnitude

$$
|A|=(4 \alpha-1)^{\frac{1}{2}} ; \quad|K|=4 \alpha-1,
$$

which increases with increasing $\alpha$. For

$$
(4 \alpha-1) / \alpha^{2}<t^{2}<4 ; \quad \alpha<\frac{1}{2}
$$

the two roots for $A$ are real, one of greater magnitude than $(4 \alpha-1)^{\frac{1}{3}}$ (but $<1$ ), the other less. Differentiating (27) gives

$$
\frac{d A}{d \alpha}=\frac{A t-2}{A-t \alpha} ; \quad A t-2<0 ; \quad A-t \alpha= \pm\left[\alpha^{2} t^{2}-(4 \alpha-1)\right]^{\frac{1}{3}},
$$

thus in the range (32) the greater of the magnitudes of the roots decreases with increasing $\alpha$. The optimum value of $\alpha$ is therefore that for which the range (30) of complex roots just covers the spectrum of $t$-values. For the largest and smallest $t$-values

$$
t= \pm[\cos (\pi / p)+\cos (\pi / q)]= \pm t_{\max }
$$

thus for $\alpha$ we use the smaller root of

$$
\alpha^{2} t_{\max }^{2}-4 \alpha+1=0 .
$$

Then for all of the error eigenfunctions

$$
|K|=K^{*}=4 \alpha-1 \text {. }
$$

For large $p$ and $q$ this may be approximated by

$$
K^{*} \cong 1-\sqrt{2} \pi\left(p^{-2}+q^{-2}\right)^{\frac{1}{2}}
$$

With this procedure and with the optimum values of $\alpha$ the number of iterations required to produce a substantial improvement in a trial solution increases about linearly with $p$ and $q$ rather than quadratically as it does for the Richardson method or the Liebmann method using $\alpha=\frac{1}{4}$. 
The usefulness of the extrapolated Liebmann method is limited by the difficulty of determining the optimum $\alpha$-value for more complex problems than the example considered here. It seems likely, however, that in many similar problems a considerable improvement in convergence rate can be achieved by a suitable choice of $\alpha$ and that an approximate optimum $\alpha$-value can be found empirically without great difficulty.

The Liebmann and extrapolated Liebmann methods have an advantage -for some types of machine applications-over the Richardson and similar methods in that they require carrying as machine "memory" no more than one complete set of $\phi_{j, k}$-values. However, for use with punched card machines or other calculating machines with severely limited internal (rapid-access) memory this advantage is offset by the difficulty of retaining the newly calculated values for use in the succeeding point and (more particularly) for the adjacent point in the succeeding row (or column). For such machines it is more convenient to use procedures in which a $\phi$-value when calculated may be stored in the "external memory" until needed in the next iteration cycle.

6. The Second-Order Richardson Method. An improvement in the convergence rate of the Richardson method comparable to that achieved for the Liebmann method by extrapolation may be gained by retaining for use in the calculation of $\phi_{j, k}^{n+1}$ not only $\phi_{j^{\prime}, k^{\prime}}^{n}\left(j^{\prime}, k^{\prime}\right.$ running over points neighboring $j, k)$ but also $\phi_{j, k}^{n-1}$. This modification is suggested by the formal equivalence between the Richardson method and the solution of the time-dependent equation, $\frac{\partial \phi}{\partial t}=L \phi$. The modified iteration method is equivalent to the solution of an equation of second order in $t$,

$$
\frac{\partial^{2} \phi}{\partial t^{2}}+A \frac{\partial \phi}{\partial t}-L \phi=0
$$

and may therefore be termed the "second-order Richardson method." The iteration process may then be written as

$$
\begin{aligned}
\phi_{j, k}^{n+1} & =\phi_{j, k}^{n}+\alpha L \phi_{j, k}^{n}+\beta\left(\phi_{j, k}^{n}-\phi_{j, k}^{n-1}\right) & & \text { (interior points) } \\
& =b_{j, k} & & \text { (boundary points). }
\end{aligned}
$$

We again denoted by $\phi_{j, k}$ the solution of the equation $L \phi_{j, k}=0$ satisfying the boundary condition incorporated in (38) and by $\epsilon_{j, k}^{n}$ the difference, $\phi_{j, k}^{n}-\phi_{j, k}$. The error then satisfies the induction equation (38) with a zero boundary condition. An error-eigenfunction, $\epsilon_{j, k}^{\nu}$, of $L$ belonging to the eigenvalue, $L_{(v)}$,

$$
\begin{aligned}
L \epsilon_{j, k}^{\nu} & =L_{\nu} \epsilon_{j, k}^{\nu} & & \text { (interior points) } \\
\epsilon_{j, k}^{\nu} & =0 & & \text { (boundary points), }
\end{aligned}
$$

is then multiplied in each iteration cycle by a factor, $K$, such that,

$$
K_{\nu}{ }^{n+1} \epsilon^{(\nu)}=K_{\nu}{ }^{n} \epsilon^{(\nu)}+\alpha L_{\nu} K_{\nu}{ }^{n} \epsilon^{(\nu)}+\beta\left(K_{\nu}{ }^{n}-K_{\nu}{ }^{n-1}\right) \epsilon^{(\nu)}
$$

or (dropping the subscript, $\nu$ )

$$
K^{2}-(1+\alpha L+\beta) K+\beta=0 .
$$


If all of the eigenvalues, $L_{\nu}$, are of the same sign (which for definiteness we take as negative) and are in the range

$$
0>L_{0} \geqslant L \geqslant L_{m},
$$

then $\alpha$ and $\beta$ can be chosen to minimize

$$
K^{*}=\max \left|K_{\nu}\right|<1 \text {. }
$$

(Since we are here using a second-order recurrence relation for $\phi^{n}$ the two roots of (40) correspond to two separate modes of decay for each eigenfunction. Both decay rates must be considered in (42).) For positive $\beta$ we again have a range of values of $\alpha L$ within which the roots of (40) are complex and of magnitude $\beta^{\frac{1}{2}}$. It may be shown, as before, that outside this range the greater $|K|$ decreases with increasing $\beta$. Thus $\alpha$ and $\beta$ should be chosen to make the range of complex roots just fit the range (41). This requires

hence

$$
\begin{aligned}
& 1+\alpha L_{0}+\beta=2 \beta^{\frac{1}{3}} \\
& 1+\alpha L_{m}+\beta=-2 \beta^{\frac{1}{1}},
\end{aligned}
$$

$$
\begin{aligned}
\alpha\left(L_{0}+L_{m}\right) & =-2(1+\beta) \\
\alpha L_{0} & =-\left(1-\beta^{\frac{1}{2}}\right)^{2},
\end{aligned}
$$

or

$$
\begin{gathered}
\eta=L_{0} /\left(L_{0}+L_{m}\right)=\frac{1}{2}\left(1-\beta^{\frac{3}{3}}\right)^{2} /(1+\beta) \\
\beta-2 \beta^{\frac{1}{2}}(1-2 \eta)^{-1}+1=0 .
\end{gathered}
$$

Choosing the smaller root gives for the decay factor,

$$
K^{*}=\beta^{\frac{1}{2}}=1-2(1-2 \eta)^{-1}\left(\eta-\eta^{2}\right)^{\frac{1}{2}} \cong 1-2 \eta^{\frac{1}{2}} .
$$

For the rectangular Laplace problem

hence

$$
\eta \cong \pi^{2}\left(p^{-2}+q^{-2}\right) / 8
$$

$$
K^{*}=1-\pi\left(p^{-2}+q^{-2}\right) / \sqrt{2} .
$$

Thus a comparison of the convergence rate of the second-order Richardson method (45) with the extrapolated Liebmann method (37) again shows a factor of two advantage over the latter.

It is not in general possible, to the writer's present knowledge, to effect a comparable further improvement in the Richardson method by using a third or higher order induction process.

7. Applications to Eigenvalue Problems. The methods here described may be used to determine the fundamental eigenfunction of an equation of the form

$$
\left(L+\lambda^{2}\right) \phi=0
$$

with homogeneous boundary conditions if the smallest value of $\lambda^{2}$ is approximately known. The operator, $L$, is replaced throughout by $L+\lambda_{0, \text { approx. }}^{2}$ and an initial trial solution chosen to approximate the fundamental eigenfunction. Then the higher eigenfunction components of the trial solution will decay during the iteration process while the fundamental remains approximately

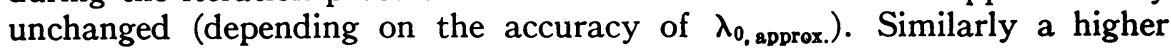


eigenfunction can be calculated by replacing $L$ by $\left(L+\lambda_{t, \text { approx }}^{2}\right)^{2}$. It is to be expected that the iteration method so obtained would be very slowly convergent since $\eta$ is the square of the ratio of the largest to the smallest value of $\left|L+\lambda_{i, \text { approx. }}^{2}\right|(i \neq 0)$.

8. Time Estimates. In order to indicate the order of difficulty of typical problems to which these methods are applicable we consider two problems; the solution of the Laplace equation and of the biharmonic equation in a square region,

$$
p=q=N \text {. }
$$

The possibility, for some forms of the boundary conditions, of treating the biharnunic equation by factoring into a Laplace and a Poisson equation, will not be considered.

We assume, for definiteness, that the iteration process is to be continued until all errors are reduced by a factor of $10^{-6}$. The number of cycles of iteration required will then be approximately,

$$
\eta_{\max } \cong 6 /\left|\log K^{*}\right|
$$

while the number of $\phi$-values to be corrected in each iteration is $(N-1)^{2}$.

We denote by $\tau$ the mean time required for each arithmetic operation. Then the time required for the evaluation of one $\phi_{j, k}^{n}$ is $T$ where

$\begin{array}{lr}\text { Laplace-Richardson } & 4 \tau \\ \text { Laplace-Liebmann ( } \alpha=\frac{1}{4} \text { ) } & 4 \tau \\ \text { Laplace-Liebmann (optimum } \alpha \text { ) } & 7 \tau \\ \text { Laplace-2nd order Richardson } & 9 \tau \\ \text { Biharmonic-Richardson } & 12 \tau \\ \text { Biharmonic-Liebmann (with extrapolation) } & 15 \tau \\ \text { Biharmonic-Liebmann (no extrapolation) } & 12 \tau \\ \text { Second-Order Richardson } & 17 \tau\end{array}$

The total calculating time is given in the following table for a $10 \times 10$ and for a $20 \times 20$ lattice, and the asymptotic form for large $N$

Approximate Total Calculating Time

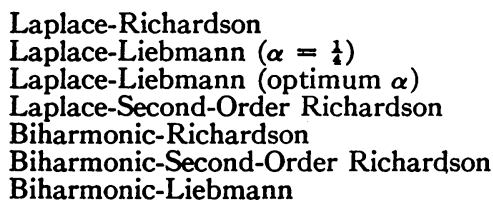

$\begin{array}{ccc}N=10 & N=20 & N \text { Large } \\ 9 \cdot 10^{4} \tau & 1.6 \cdot 10^{6} \tau & 11 N^{4} \tau \\ 5 \cdot 10^{4} \tau & 8 \cdot 10^{5} \tau & 5.6 N^{4} \tau \\ 1.2 \cdot 10^{4} \tau & 1.1 \cdot 10^{5} \tau & 15 N^{3} \tau \\ 3 \cdot 10^{4} \tau & 3 \cdot 10^{5} \tau & 40 N^{3} \tau \\ 1.1 \cdot 10^{7} \tau & 8 \cdot 10^{8} \tau & 14 N^{6} \tau \\ 4 \cdot 10^{5} \tau & 7 \cdot 10^{6} \tau & 48 N^{4} \tau \\ ? & ? & ?\end{array}$

For the biharmonic equation the ratio, $\eta=L_{0} /\left(L_{0}+L_{m}\right)$, has been approximated by the square of the corresponding value for the Laplace equation.

To convert these values to true time estimates we may, quite crudely, ?pproximate $\tau$ by $10^{-5}$ days for electromechanical computers (e.g., punched card machines) and by $10^{-8}$ days for entirely electronic computers. It is thus seen that with a fairly fine mesh the calculating time required with the slower machines is uncomfortably large for the Laplace equation and prohibitive for the biharmonic equation if the normal Richardson method is 
used. Even with the faster machines the time required for the solution of a biharmonic equation by the methods considered here is uncomfortably large unless the second order Richardson (or probably also the extrapolated Liebmann) method is used. It is clear that for many problems of interest the simplest iterative procedures will prove impossibly tedious even with the fastest automatic computers.

The apparent likelihood that the extrapolated Liebmann procedure would prove more rapidly convergent and more convenient for electronic computers than the second-order Richardson method would seem to justify an experimental study with such a computer.

The writer is indebted to R. H. MacNeal and W. E. Milne for encouragement and helpful discussion.

California Institute of Technology

\section{Stanley P. Frankel}

Pasadena, California

${ }^{1}$ D. R. HARTREE, "The ENIAC an electronic computing machine," Nature, v. 158, 1946, p. 500-506. [MTAC, v. 2, p. 222]

IBM Automatic Sequence Controlled Calculator, IBM Corporation, New York, 1945.

ANON., "The UNIVAC," Electronic Industries, v. 2, 1948, p. 9.

H. H. Goldstine \& J. von Neumann, Planning and Coding of Problems for an Electronic Instrument, Institute for Advanced Study, Princeton, 1947. [MTAC, v. 3, p. 54-56]

$2 \mathrm{R}$. V. SouTHWELL, Relaxation Methods in Engineering Science, Oxford, 1940. 1932.

${ }^{3}$ E. T. WhitTAKer \& G. Robinson, The Calculus of Observations, London and Glasgow,

¿ G. Shortley, R. Weller, \& B. Fried, “Numerical solution of Laplace's and Poisson's equations with applications to photoelasticity and torsion," Ohio State University, Studies, Engineering Series, Bull. no. 107, 1942.

5 L. F. RICHARDSON, "The approximate arithmetical solution by finite differences of physical problems involving differential equations, with an application to the stresses in a masonry dam," R. Soc., London, Phil. Trans. s. A, v. 210, 1911, p. 307-357. "How to solve differential equations approximately by arithmetic," Math. Gazette, v. 12, p. 415-421, 1925.

' R. Courant, "Uber partielle Differenzengleichungen," Congresso Internazionale dei Matematici, Atti, Bologna, v. 3, 1930, p. 83-89.

7 This can be shown by the method used by H. LEwy in "On the convergence of solutions of difference equations," Studies and Essays Presented to R. Courant on his 60th Birthday, New York, 1948, p. 211-214.

8 H. LiEBMANN, "Die angenäherte Ermittelung harmonischer Funktionen und konformer Abbildungen," Bayer. Akad. Wiss., math.-phys. Klasse, Sitz., 1918, p. 385-416 Further references to the Liebmann method are cited in footnote 1 of Shortley, Weller \& Fried. See also $M T A C$ v. 3, p. 350, footnote 3 .

\section{On a Definite Integral}

The function

$$
f(x)=\int_{0}^{\infty}(u+x)^{-1} \exp \left(-u^{2}\right) d u
$$

is tabulated by E. T. Goodwin and J. Staton ${ }^{1}[M T A C$, v. 3, p. 483] from series expansions and by numerical integration of the differential equation satisfied by the function.

The integral can be evaluated explicitly in terms of two simple tabulated functions. The writer used the LAPLACE transform ${ }^{2}$ to evaluate the integral. This method will now be given.

We may write

$$
I(t)=\int_{0}^{\infty}\left(u+t^{\frac{1}{3}}\right)^{-1} \exp \left(-u^{2}\right) d u=\int_{0}^{\infty}(1+v)^{-1} \exp \left(-t v^{2}\right) d v
$$

